https://doi.org/10.48009/1_iis_2014_52-60

Issues in Information Systems

Volume 15, Issue I, pp. 52-60, 2014

\title{
ORGANIZATIONALLY AGNOSTIC BUSINESS MODELING: HOW TO MAKE BUSINESS ARCHITECTURE ADAPTABLE TO ORGANIZATIONAL CHANGE
}

\author{
Carlos E. Martinez, The MITRE Corporation, cmartinez@mitre.org \\ Sheila A. Cane, The MITRE Corporation, sheila@mitre.org
}

\begin{abstract}
This paper presents a practical approach to improving the business processes of an enterprise independent of its organizational structure. The objective of the approach is to enable discovery of potential duplications of effort or inconsistencies in organizational responsibilities among the organizations that comprise the enterprise. Standard modeling techniques are incorporated to provide a step-by-step approach an organization can easily implement. The approach, which builds upon prior work by the authors, was developed to support the needs of a government organization that had undergone a significant reorganization and needed to determine if its new organizational structure adequately met its mission responsibilities.
\end{abstract}

Keywords: Business Process Modeling, IDEF0 Activity Model, Swim Lanes, Organizational Change

\section{INTRODUCTION}

Almost every enterprise is affected by continually changing political, operational, environmental, and technical (POET) factors. Organizations must be able to operate within these changing environments. Strategic use of enterprise architecture methods can enable the organization to effectively change according to its needs. However, while considerable work has been done developing business architecture methods, there are few descriptions of practical applications on how multiple techniques can be used together.

This paper demonstrates how activity and swim-lane models can be used to model top-level operational processes in a way that enables organizational change; it describes a prescriptive approach to modeling an organization's business processes independently of the allocation of its activities to subordinate components. It describes the approach including the models used for each stage of the process.

The approach had its preliminary origins in work done in the late 1990s in support of defining architectural approaches for the Department of the Army. [4] The approach was then subsequently generalized in 2002 first for the U.S. Air Force [3], then for overall application to the Department of Defense [2] and more formally codified thereafter [6]. The current authors have built upon the earlier conceptual work to develop a more practical approach in support of a government agency. MITRE's work on this effort was provided under the sponsorship of the Department of Homeland Security. The work demonstrated in this paper was the result of the collaboration of The Homeland Security Systems Engineering \& Development Institute (HS SEDI) operated by the MITRE Corporation and Department of Homeland Security (DHS) enterprise architecture personnel.

\section{Business Modeling Approach}

Activity models can independently show inputs, outputs, actors, and constraints. Swim lane diagrams can show organizational or functional relationships and information flow. Using these types of models enables an organization to review operational processes, identify gaps and duplication of effort, and make organizational changes as required, e.g., reassign activities from one organization to another, add new activities, or discontinue activities.

The recommended approach to describing a business area independent of organizational structure is to describe it in terms of the business activities that the overall organization needs to accomplish. This is best done by using the 
IDEF0 [5] modeling technique to systematically decompose the organization's business activities in a series of progressively more detailed levels down from a single high level that describes all of the outputs of the organization, all of the inputs required to produce those outputs, the governing laws, regulations, plans, etc. that prescribe or control the execution of the activities, and the mechanisms (in this case the organizations or actors) that perform the activities.

The use of this approach enables the definition of the activities independently of the organizations that conduct them because the organizations are identified only after-the-fact as one of the mechanisms that enable the activities. After the activity models are developed, swim lane diagrams are used to show the lowest level activities and information flows across organizations as they currently exist. The swim lane diagrams will help the organization review and analyze the current state with stakeholders to create a more effective future state. While the method should be followed sequentially, it is structured so that one can go back and forth between steps as knowledge and understanding about the organization are gained.

As presented in Figure 1, the IDEF0 business modeling approach calls for explicitly identifying five artifacts:

1. The Activity being accomplished

2. The Inputs to the activity

3. The Controls that govern how the activity should be performed

4. The Outputs from the activity

5. The Mechanisms that enable the activity to be performed

\section{Based on Activities and ICOMs (Inputs, Controls, Outputs, Mechanisms)}

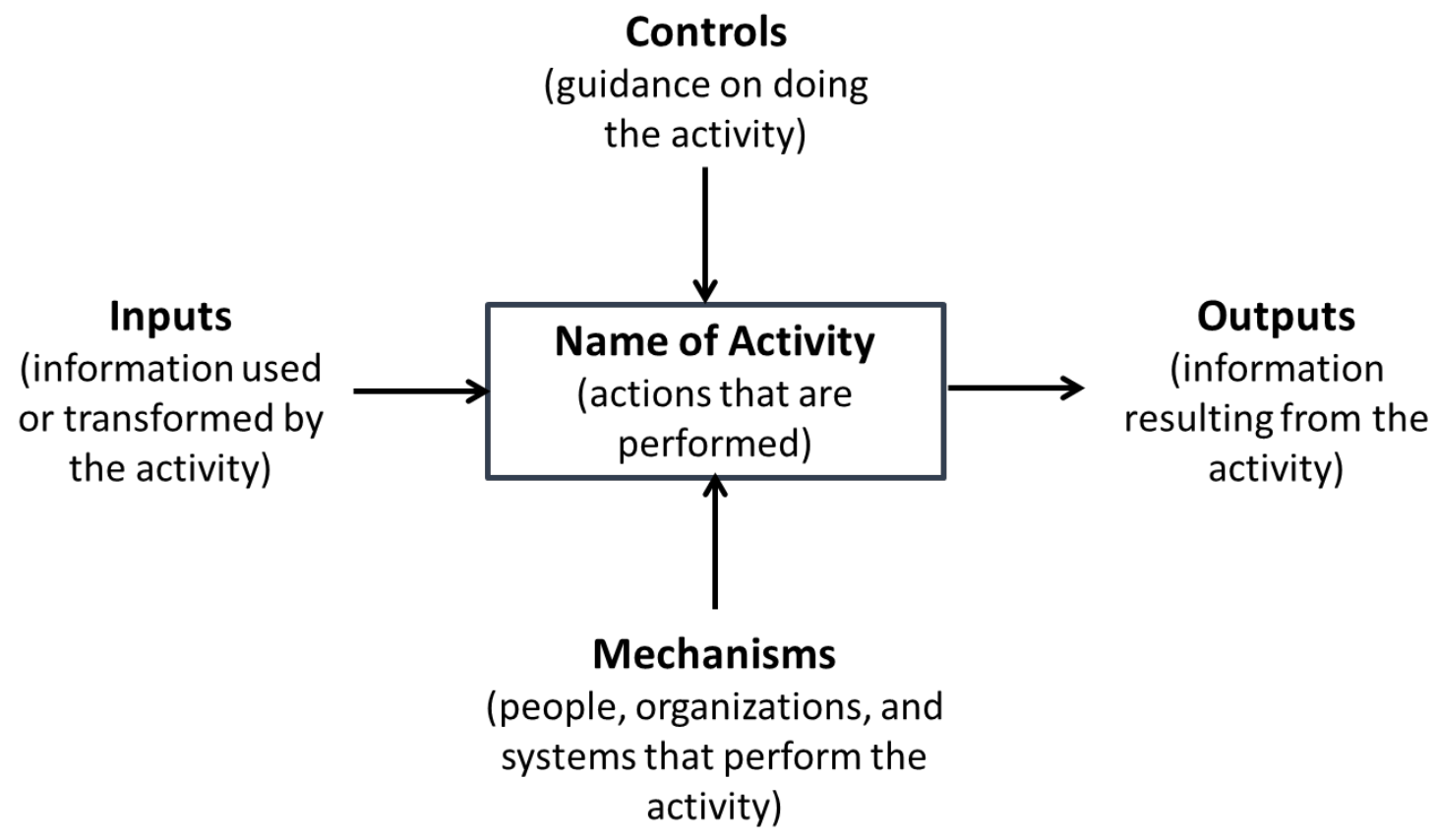

Figure 1. IDEF0 Business Modeling Approach 
Collectively, the Inputs, Controls, Outputs, and Mechanisms are called ICOMs. ICOMs should first be identified at the highest level and then progressively refined at lower levels of detail through hierarchically decomposing activities into sub-activities. Mechanisms may consist of organizations (actors) and/or the systems used by the actors to accomplish the activity.

\section{Detailed Modeling Approach}

\section{Step 1. Development of Activity Model}

The first step is to identify the ICOMs at the highest level of the business (the A-0 level). One should start with defining the outputs (or products of the business), which for many business functions often consists of information produced in various forms such as reports, briefings, notices, etc. Next, one defines the inputs, again usually in the form of different types of data or other information, needed to generate the outputs. Then, one should identify the principal controls (laws, regulations, executive orders, plans, etc.) that govern why, when, and how the activities should be conducted. Finally, the appropriate mechanisms (mostly in terms of responsible organizations), should be identified for the totality of the activities conducted.

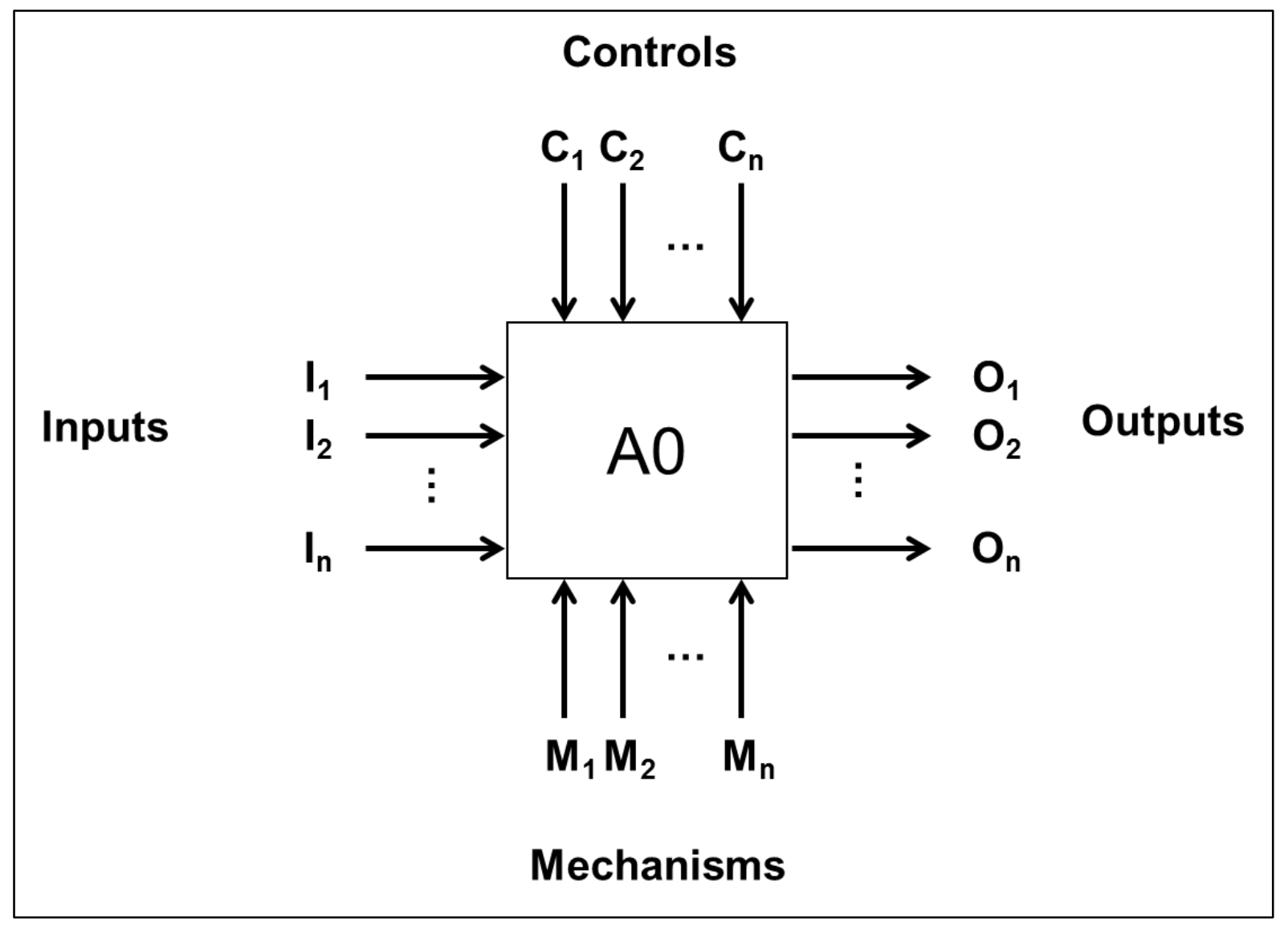

Figure 3. Identify ALL the ICOMs at the Highest Level

The value of starting at the top-level is that it presents an organizationally agnostic view of the business. This feature begins to come into view at the first level of decomposition of the overall business into major business functions.

To build the IDEF0 activity model, one has to hierarchically decompose the highest-level activity into subordinate sub-activities, which in turn may even be further broken down. To ensure common understanding among business 


\section{Issues in Information Systems \\ Volume 15, Issue I, pp. 52-60, 2014}

modelers, the IDEF0 methodology employs a standardized activity numbering convention, wherein the top-level activity is defined as the "zero" level, or A0. The first-level set of major activities is numbered A1, A2, A3, etc. Further refinement of these activities into sub-activities results in sub-activities numbered A11, A12, A13, etc.; decomposing A12 continues with A121, A122, etc. This convention, formally defined in FIPS 183 [5], makes it easy to identify the "parent" activity for any subordinate level.

An example activity hierarchy is shown in Figure 2. It should be noted that not all activities need to be decomposed to the same level of detail. One should conduct the decomposition only down to a level that will help answer questions of interest about the business processes.

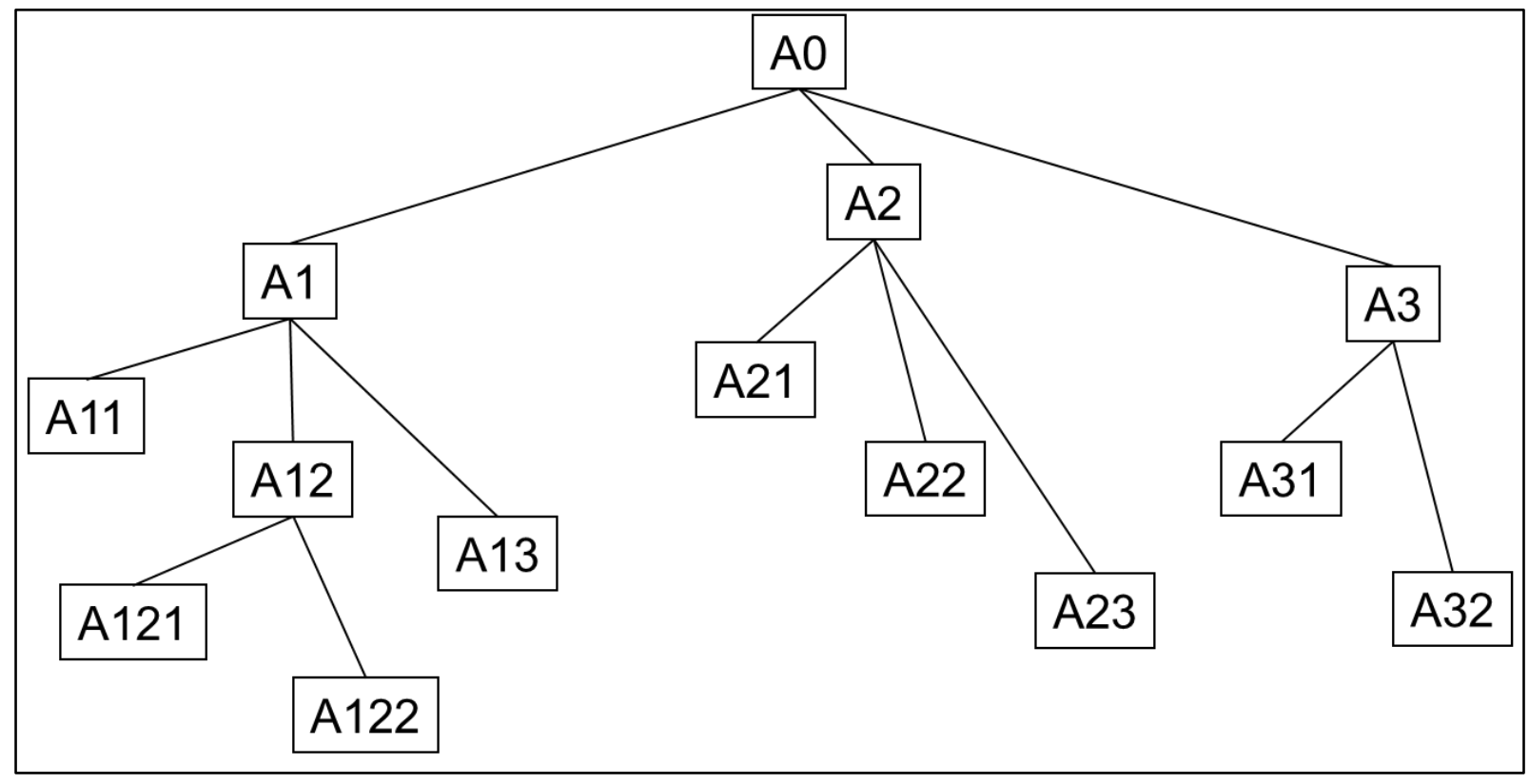

Figure 2. Build a Hierarchy of Activities 


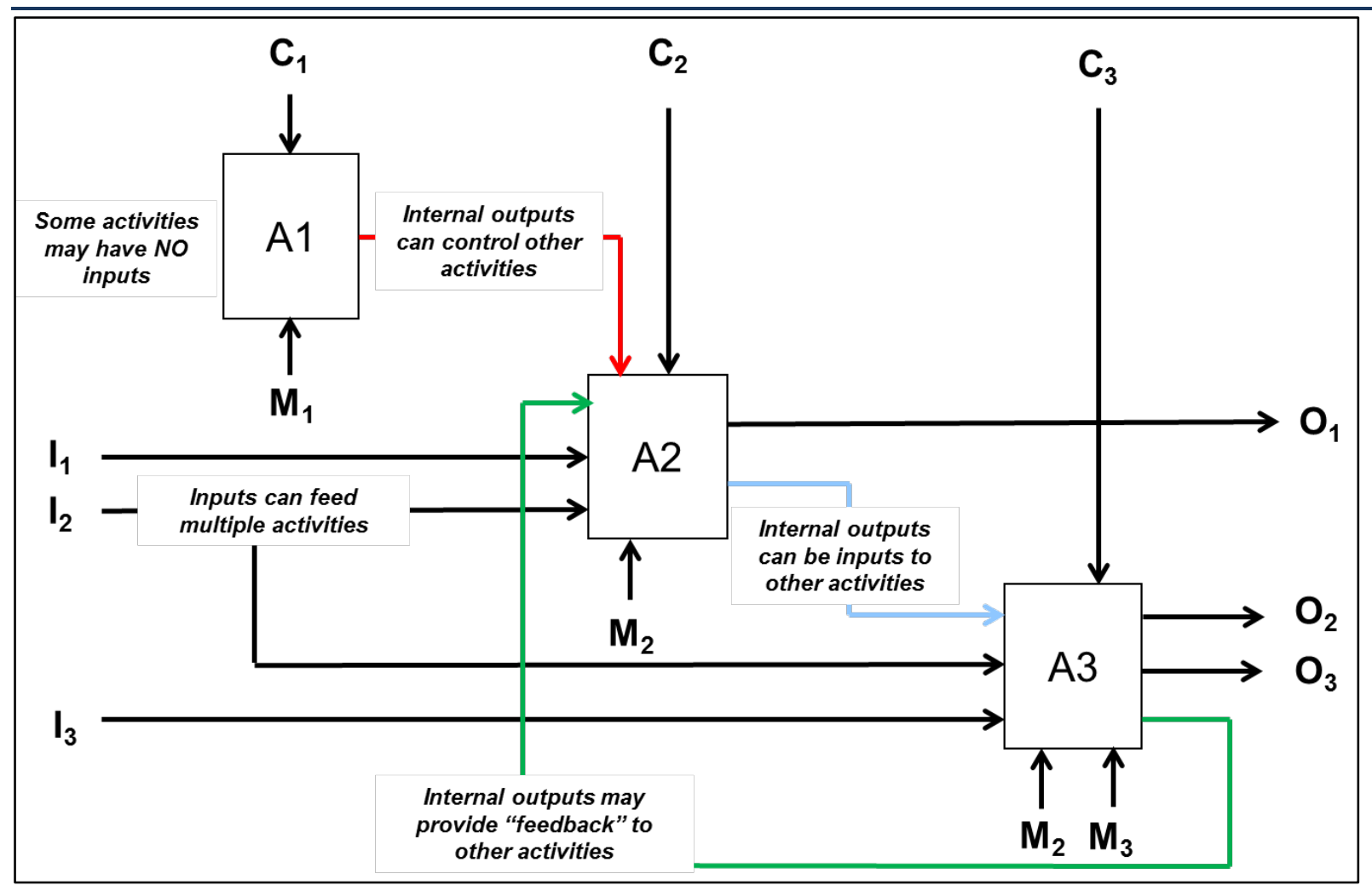

Figure 4. Define ICOMs for Lower Activities

As shown in Figure 4, all of the ICOMs depicted on the parent (A0) diagram show up at this level of decomposition, but have now been allocated to various major business functions (A1, A2, and A3). At this stage there are other aspects of the IDEF0 business modeling approach that are worth noting:

1. Every activity should have an output. This should make intuitive sense, because if an activity does not produce any output, it does not add any value and probably should not be done.

2. Every activity should have a control that governs why, when, or how to do it.

3. Not every activity needs an input. For example, the sole purpose of activity A1 in this diagram is to respond to a governing control (C1) to develop a plan (its output) on how Activity A2 should be performed. So, this is a case of an activity whose output does not show up on the parent level (A0) diagram because its purpose is an internal one. And, its output serves as a control on another internal activity--how A2 is performed.

4. Some inputs can serve as inputs to more than one activity. In this diagram, for example, I2 serves as an input to both $\mathrm{A} 2$ and $\mathrm{A} 3$.

5. Some outputs serve simply as internal inputs to other business activities, such as the output line from A2 that is used as a subsequent input by $\mathrm{A} 3$, or the output from A3 that serves as a feedback input for later iterations of A2.

6. Finally, every activity should have an identified mechanism to perform it. As seen in this diagram, some mechanisms such as M2 perform activities A2 and A3, while some activities require more than one mechanism to conduct it such as A3 which requires support from M2 and M3.

It is at this level that the organizationally agnostic value of the IDEF0 methodology should become apparent. The organizations performing the work are captured as mechanisms because the work is the work, regardless of who does it. 


\section{Issues in Information Systems \\ Volume 15, Issue I, pp. 52-60, 2014}

Because activity $\mathrm{A} 3$ results in more than one output $(\mathrm{O} 2$ and $\mathrm{O} 3)$ and is performed by more than one organization (M2 and M3), it is probably a fairly complex activity that should be further decomposed to a more detailed level. Figure 5 shows further decomposition of activity A3 into two sub-activities, A31 and A32. At this level, one can see that the output from $\mathrm{A} 2$ is used by M2 to produce output $\mathrm{O} 2$ by performing activity $\mathrm{A} 31$ and that inputs $\mathrm{I} 2$ and $\mathrm{I} 3$ are used by M3 to produce output $\mathrm{O} 3$ by performing A32. And, that from its work on A32, M3 provides feedback to A2.

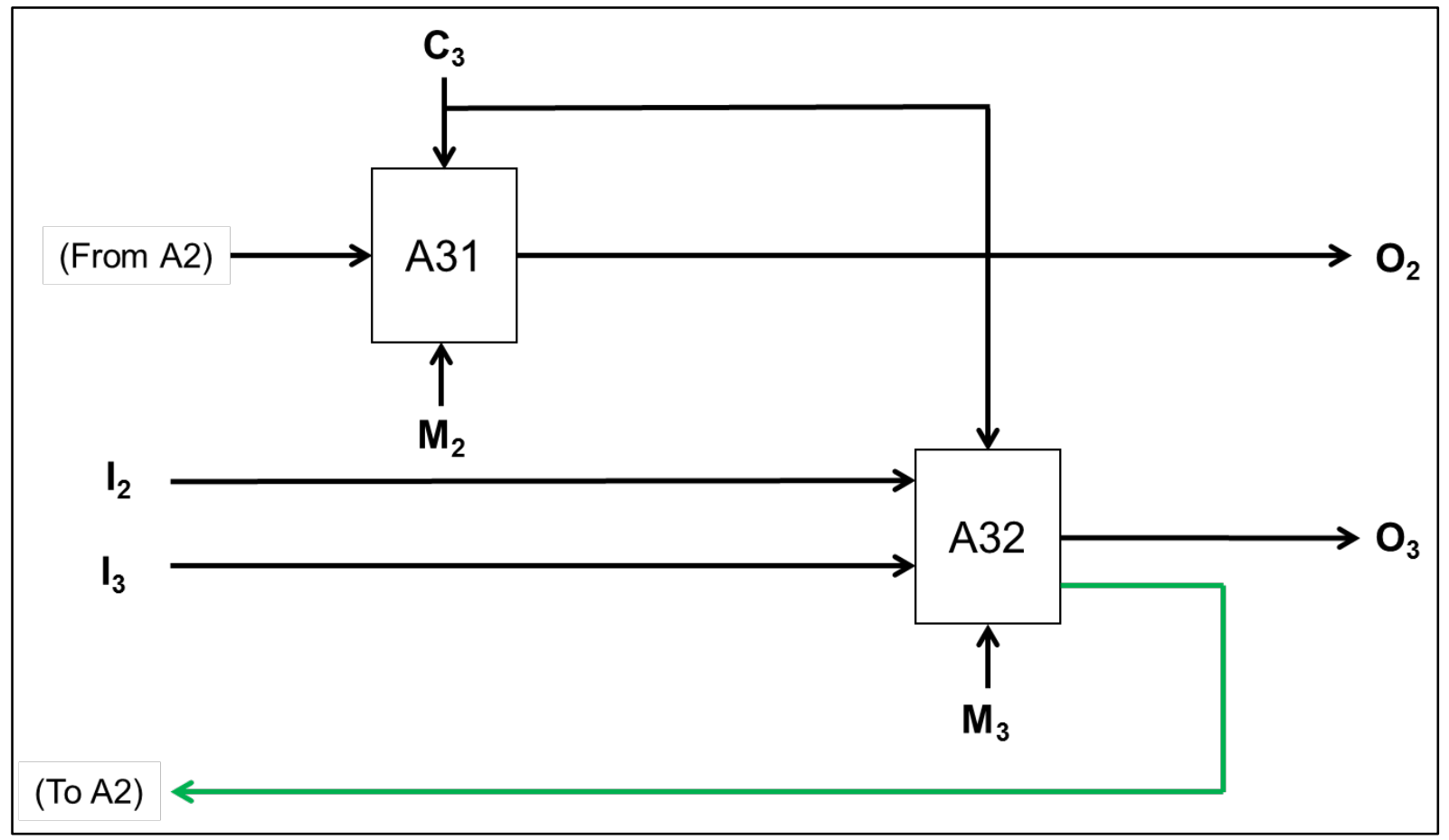

Figure 5. Continue Decompositions until Activities Are Performed by Only One Organization

One could continue decomposing activities A31 and A32 even further to examine their details, such as to determine how $\mathrm{I} 2$ and $\mathrm{I} 3$ are used to produce $\mathrm{O} 3$ and the feedback to A2. However, because the objective of this sample analysis is only to determine the allocation of functions between M2 and M3, one can stop at this point of the decomposition unless it subsequently becomes necessary for more detailed analysis.

\section{Step 2. Identification of Leaf-Level Activities}

Having decomposed all of the activities down to the lowest level of interest (e.g., being performed by only one organization), the next step is to identify the lowest level of activities within the hierarchy, as depicted in Figure 6 . These lowest level activities, each performed by an individual organization, can be linked to show the sequential processes by which the organization produces the overall business results. 


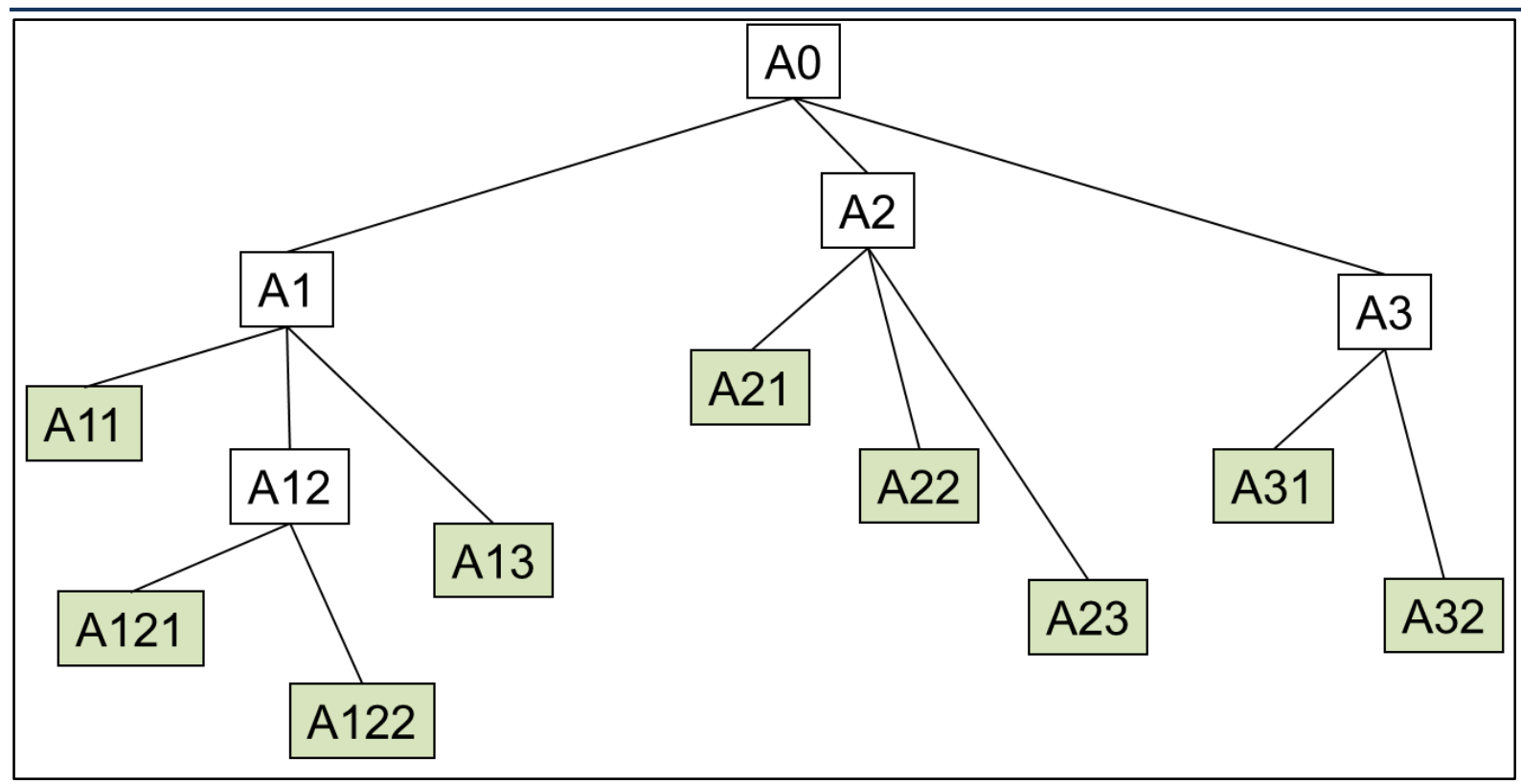

Figure 6. Identify Lowest (Leaf-level) Activities

Because the IDEF0 methodology represents all of the activities performed by the organization, one has to first define a particular subset of activities for which one can define a sequential set of activities that provide the "thread" to link the activities into a process flow. This is often the most difficult task to accomplish in that it requires considerable effort to ensure that all of inputs and outputs are adequately captured in a flow-process order. In doing so, it is of particular importance to identify backward flows that represent feedback from one activity to another.

\section{Step 3. Arranging Leaf Level Activities into Swim Lanes}

To see the information flows within and between organizations, the "thread" of information inputs and outputs is best presented in terms of "swim lanes" wherein the activities conducted are aligned by organization and sequenced to show the flow from inputs, through activities, to outputs. The swim lane diagram, shown in Figure 7, is particularly useful for identifying cross-organizational issues such as duplication of effort, and gaps in understanding of information needs and flows. 


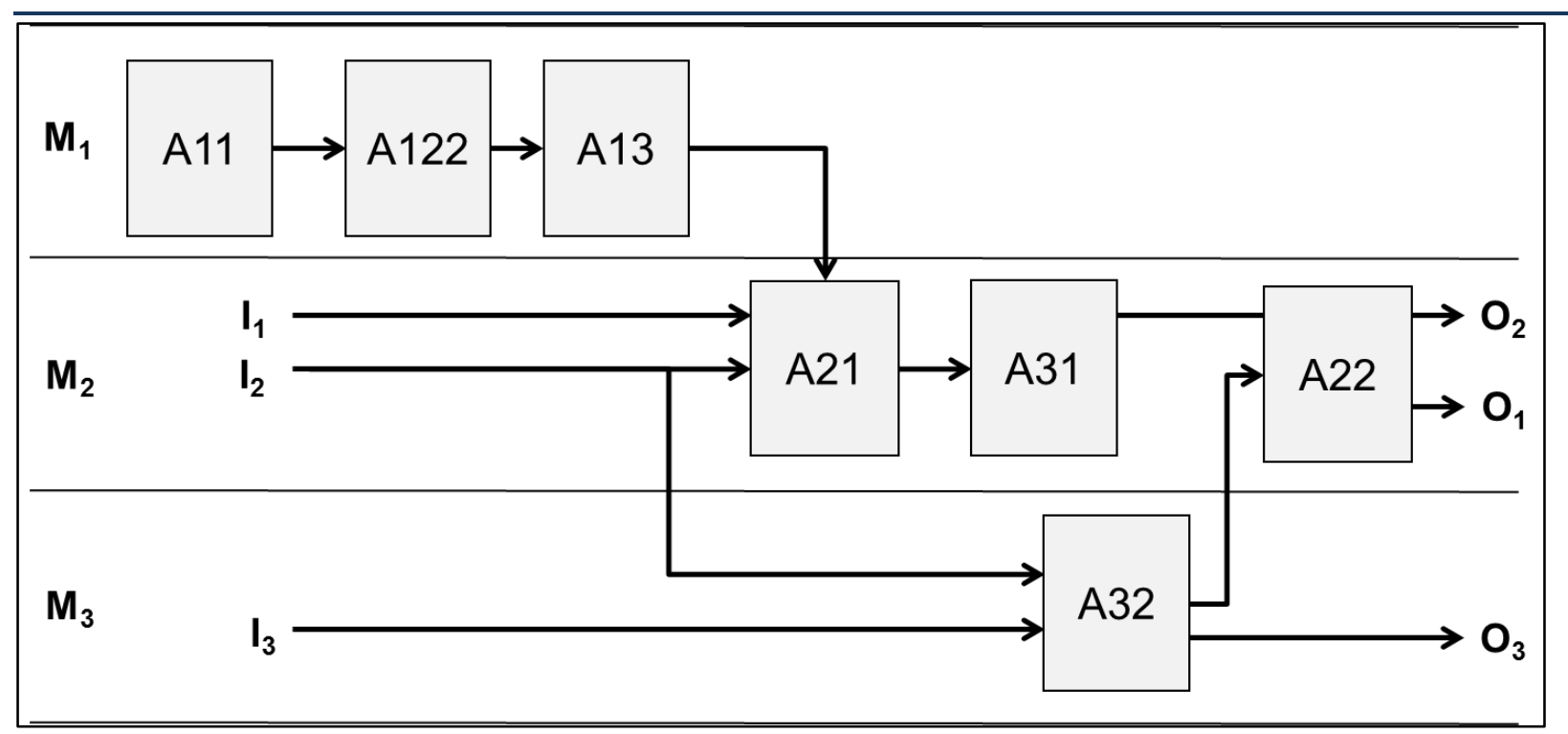

Figure 7. Define "Swim Lanes" for the "Mechanism" Organizations to Show Information Flow

Because the IDEF0 modeling approach focuses first on what is done and not on who does it, it is organizationallyagnostic. As such, the approach can be focused on the current (As-Is) business processes of an organization to identify potential duplications of effort as well as gaps (i.e., activities that either are not being done, or for which no specific organizations can be identified as responsible). The same approach can be also be used to define new ways of doing the work and, based on the distribution of appropriate skills and capabilities, which organizations should do it (the To-Be).

\section{Application of Approach}

The organizationally agnostic approach was successfully applied by the authors to analyze a U.S. Government organization whose organizational structure had evolved over time but whose overall mission responsibilities had not changed. The application of the approach, particularly the visual representations provided by the swim lanes, demonstrated where opportunities were available to conduct realignment of responsibilities for an organization within the U.S. Department of Homeland Security [1]. This approach can be easily used by any organization undergoing change. It can facilitate understanding of activities, responsibilities, and relationships among the parties to enable a more effective organizational design.

\section{CONCLUSIONS}

In conclusion, by first defining the business functions independently of the organizations that perform them and then associating the organizations with the activities as mechanisms that enable their performance, one can readily identify opportunities for business improvement in terms of either streamlined processes or realigned organizational responsibilities.

\section{Acknowledgments}

This paper was written by the MITRE Corporation, in collaboration with and funding from the Deputy Assistant Secretary of Homeland Security, Cybersecurity \& Communications (CS\&C), through the Department of Homeland Security FFRDC (Homeland Security Systems Engineering and Development Institute (HS SEDI)) under contract \# HSHQDC-09-D-00001. 


\section{Issues in Information Systems}

Volume 15, Issue I, pp. 52-60, 2014

\section{REFERENCES}

1. Cane, S.A. and C.E. Martinez, Organizationally-Agnostic Business Modeling: A Case Study, Issues in Information Systems, Vol. 13, Issue 2, pp. 404-410. International Association for Computer Information Systems, 2012.

2. Coffin, J.S. and C. E. Martinez, ACS Architecture Development Methodology (Unpublished Briefing). ACS Defense, Inc. August 2, 2002.

3. Martinez, C.E., Developing Operational Architectures (Unpublished Briefing). Electronic Systems Center, United States Air Force. June 18, 2002.

4. Martinez, C.E., J.S. Coffin, et. al., Army Enterprise Architecture Framework Document, Version 1.0. Developed for the U.S. Army Office of the Director of Information Systems for Command, Control, Communications, and Computers by Betac Corporation, Alexandria, Virginia. September 30, 1997.

5. Federal Information Processing Standard (FIPS) Publication 183 (Draft), Integrated Definition for Function Modeling (IDEF), National Institute of Standards and Technology (NIST). December 21, 1993. (Note: The document was withdrawn as a mandated Federal standard by NIST on September 8, 2002 in accordance with the requirements of the National Technology Transfer and Advancement Act of 1995 (P.L. 104-113), because it duplicated the industry standard 1320.1-1998 - IEEE Standard for Functional Modeling Language - Syntax and Semantics for IDEF0.)

6. Ring, J.R. and D. Nicholson, Activity-Based Methodology for Development and Analysis of Integrated DoD Architectures, The MITRE Corporation, McLean, Virginia. 2007. 